\title{
Introduction to magnetic resonance imaging
}

\author{
DAVID A. GIFT, MS \\ ABRAHAM PERA, DO \\ JAMES B. MOORE, MD
}

\begin{abstract}
Magnetic resonance imaging (MRI) has become an important diagnostic tool with a wide variety of clinical applications. This article provides a qualitative description of physical principles of MRI fundamental to an appreciation of the capabilities and limitations of this complex technology. The same physical principles are used to explain the appearance of the images and the ability of MRI to provide spectroscopic data. Contrast agents, types of MRI equipment, and the safety of MRI in carrying out patient population goals are discussed. This article provides background knowledge for two subsequent articles which cover clinical application of MRI in greater detail.
\end{abstract}

Magnetic resonance imaging (MRI) has become an important tool for imaging the brain and spinal cord, and its role in imaging other parts of the body is rapidly becoming more significant. The basic principles of MRI image production, as well as its appearance, types of magnets used, spectroscopy, hazards, and contraindications will be discussed in this article, which is the first of a series of three. In the second and third, we will discuss indications for MRI of the body and CNS, respectively.

In general, a diagnostic image is a representation of a physical property or properties of the substances within the field of view. In a photograph, the physical property is reflected light, in computed tomography (CT), it is x-ray absorption, in ultrasonography, it is reflected sound, and in MRI, it is net magnetization.

The following description of the physical principles of magnetic resonance is intentionally funda- mental. It is intended to provide sufficient knowledge to assist the reader in evaluating and incorporating the volume of literature regarding MRI. The five articles in the reading list offer comprehensive treatment of this subject.

\section{Physical principles}

The nuclei of certain elements have a nonzero magnetic moment. (Hydrogen has this property and is used for imaging.) One may think of nuclei with this property as behaving like small bar magnets. The net magnetization of a sample (for example, a volume of tissue) is equal to the sum of these individual nuclear magnetic moments. In the absence of a strong magnetic field, the nuclear magnetic moments are oriented randomly, and the sample net magnetization is zero. When an external magnetic field is applied to the sample, the nuclear magnetic moments will tend to align themselves with the field in an equilibrium distribution. The net magnetization of the sample then may be described by a vector pointing in the direction of the applied magnetic field (the longitudinal direction), with its length proportional to the density of nuclear magnetic moments in the sample and to the strength of the applied magnetic field (Fig 1).

The nuclear magnetic moments never align perfectly with the applied magnetic field. Consequently, a torsional force causes them and the net magnetization vector to precess (spin or wobble) about the longitudinal axis of the applied field (Fig 2 ). The rate of precession is proportional to the strength of the applied magnetic field. Furthermore, each nuclear species (for example, hydrogen, phosphorus, fluorine) has a unique proportionality constant. The rate of precession of a given nuclear species in a given magnetic field strength is called its resonance frequency. 


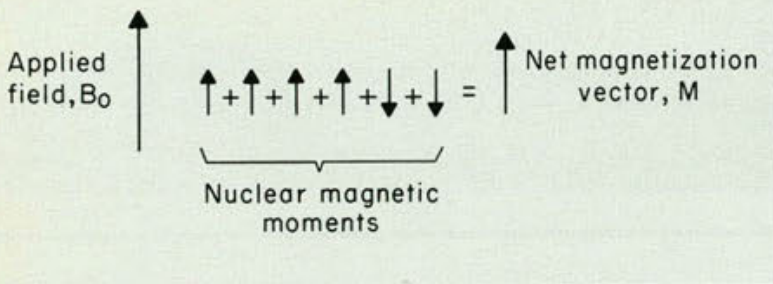

Fig 1. When nuclear magnetic moments are exposed to an applied magnetic field, they align themselves with the field. The sum of the individual nuclear magnetic moment vectors is a vector that describes the net magnetization of the sample.

A resonance condition is one in which energy may be transferred to and from a system very efficiently under unique conditions. Under any other conditions, energy transfer does not occur. In the case of nuclear magnetic resonance, nuclei may absorb energy in the form of electromagnetic waves only when the frequency of the waves exactly matches the nuclear magnetic resonance frequency. In the precessing nuclear magnetic moments model used here, this is because the oscillations of the magnetic component of the waves are coupled exactly to the precessional motion of the nuclear magnetic moments. Under typical MRI conditions, the resonance frequency of elements of biologic interest, especially hydrogen, is in the $\mathrm{MHz}$ (radiofrequency) range.

Nuclear magnetic moments exposed to radiofrequency (RF) electromagnetic waves experience additional torque from the magnetic component of the waves. This torque may be used to rotate the nuclear magnetic moments away from their align- ment with the applied magnetic field. If sufficient $\mathrm{RF}$ energy is delivered to a sample to rotate its net magnetization vector by 90 degrees, it is said that a 90-degree pulse of RF energy has been used.

Immediately following a 90-degree pulse, the net magnetization vector will be precessing in a plane perpendicular to the direction of the applied magnetic field. That is, it will have been given a transverse orientation (Fig 3). In this orientation, the precessing net magnetization vector of the sample and the applied magnetic field interact to produce an $\mathrm{RF}$ signal at the resonance frequency. When the external source of RF energy is switched off, the nuclear magnetic moments in the sample relax back to their equilibrium orientation (that is, parallel alignment) with the applied magnetic field. As this happens, the transverse component of the net magnetization vector shrinks, and the strength of the RF signal emitted by the sample decays away. The emitted signal is received by the scanner, and provides the data used to produce images.

The resonance frequency of a nucleus is much more strongly dependent on the nuclear species than it is on the strength of the local magnetic field. Thus, one can purposely manipulate local magnetic field strength to achieve certain imaging objectives without running the risk of having the resonance frequencies of the nucleus of interest (hydrogen) overlap with the resonance frequencies of nuclear species that are not of interest. Gradient magnetic fields may be applied to artificially create different magnetic field strengths across the patient's body. The dependency of resonance frequency on field strength then may be used for slice selection and for encoding the spatial location of the source of emitted MRI signals.

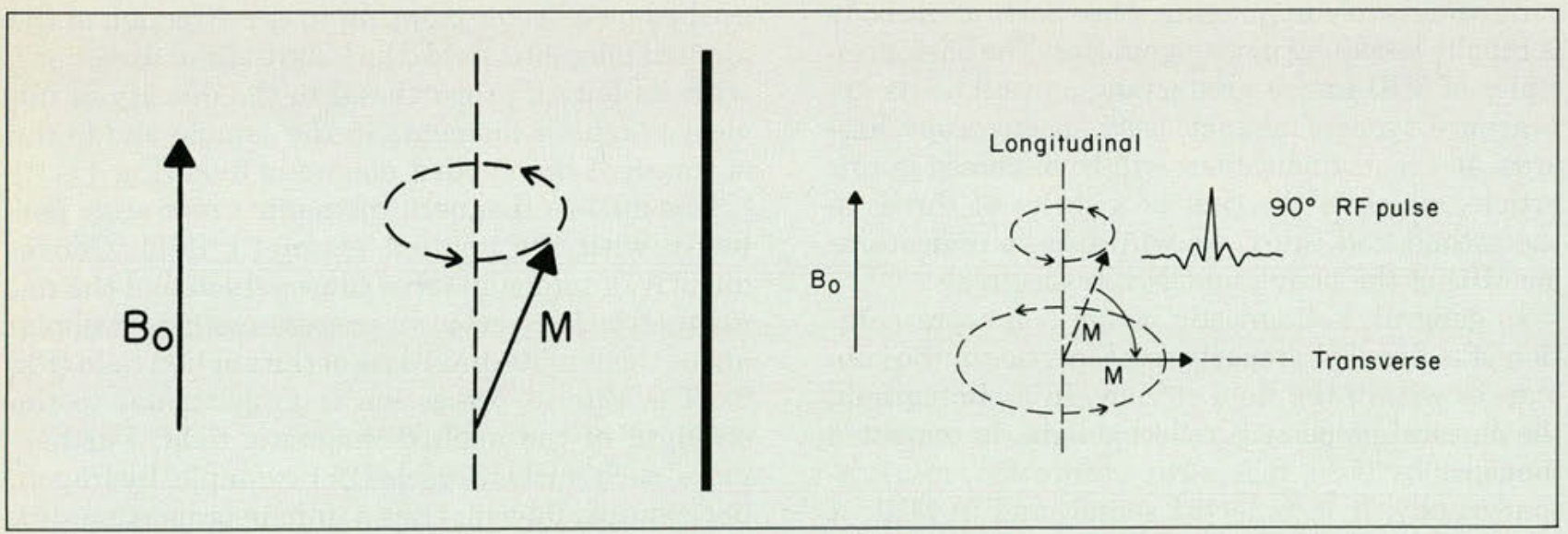

Fig 2.(left) Because nuclear magnetic moments do not align perfectly with the applied field, the net magnetization vector is forced to precess about the longitudinal axis. Fig 3.(right)The right amount of radiofrequency energy (whose frequency is matched exactly to the precessional frequency of $\mathrm{M}$ ) can tip M 90 degrees away from the direction of $B_{0}$, so that $M$ will precess 90 degrees to $B_{0}$ in the transverse plane. 


\section{In the transverse plane:}
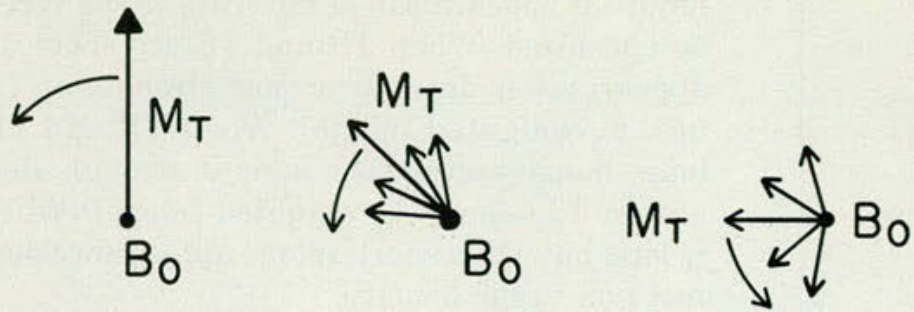

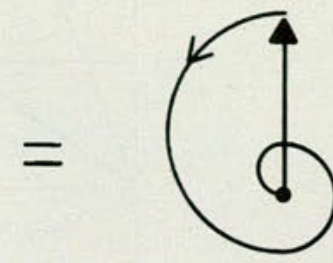

Net size and direction of vector

\section{Time}

Fig 4. Loss of transverse magnetization $(M)$ because of " $T_{1}$ relaxation." As $M$ realigns with $B$ following a 90 -degree radiofrequency pulse, the transverse component of $M, M_{T}$ shrinks. Because it is $M_{T}$ that produces the emitted RF signal, the signal decays away as relaxation occurs.

Manipulation of gradient magnetic fields also may be used to enable the simultaneous acquisition of image data from multiple slices, and to provide direct imaging capability in transaxial, sagittal, and coronal planes. In fact, many commercial MRI systems can directly produce images in any arbitrarily defined oblique plane as well. Because the image data are acquired directly in MRI, spatial resolution is equally good regardless of the orientation of the imaging plane. This differs, for example, from CT, where the spatial resolution of coronal or sagittal images may be degraded by the need to reformat image data originally acquired in the transaxial direction.

The decay of the emitted RF signal is characterized by two time constants, $\mathrm{T}_{1}$ and $\mathrm{T}_{2}$. These describe the loss of signal strength by two processes. The rate at which the net magnetization returns to its equilibrium orientation is described by $T_{1}$ (Fig 4). $\mathrm{T}_{2}$ describes the rate at which transverse magnetization is lost by a more subtle means. Recall that the magnetic resonance frequency of a nucleus is proportional to the strength of the magnetic field in which it resides. Because the applied magnetic field is not perfectly uniform, some hydrogen nuclei will experience a field strength that is different from that experienced by other hydrogen nuclei, and the precessional frequencies also will differ.

In addition, very little hydrogen exists as a free element in the body. Most hydrogen atoms are bound in the molecular structures of various chemical compounds. Moving charged particles, such as the electrons orbiting atomic nuclei, produce a small magnetic field of their own. These electronic magnetic fields offset or modify the large magnetic field applied to a sample during nuclear magnetic resonance observation. Further, orbiting electrons are not distributed uniformly about a molecule, so the hydrogen nuclei bound in different positions in different molecules will experience slightly different magnetic field strengths and have slightly different resonance frequencies also.

Because the individual nuclei in the sample have slightly different resonance frequencies, they are not precessing at exactly the same rate. Thus, immediately following a 90-degree RF pulse, the individual magnetic moments will begin to lose phase with each other and will spread out in the transverse plane (Fig 5). $\mathrm{T}_{2}$ describes the rate at which net transverse magnetization of the sample is lost because of this loss of phase between the individual nuclei contributing to the sample net magnetization.

Obviously, $T_{1}$ and $T_{2}$ will be determined to a great extent by the biochemical properties of the substance under observation. The superior tissue contrast of MRI results from the fact that the images depend upon $\mathrm{T}_{1}$ and $\mathrm{T}_{2}$ in addition to density (D). Image appearance also depends on the way in which the net magnetization of the substances in the field of view is manipulated by the applied RF pulses. To illustrate this, we consider the parameters that characterize the most popular pulse sequence used to produce clinical MR images, the spin-echo (SE) sequence. 


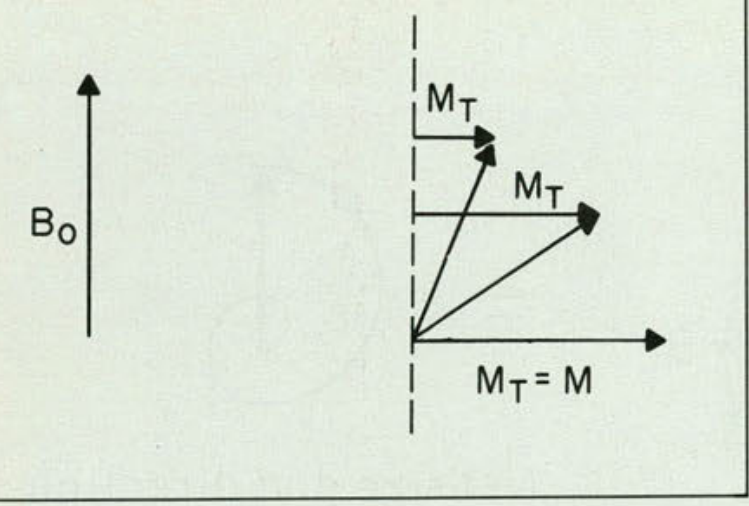

Fig 5. The individual nuclear magnetic moments precessing in the transverse plane following a 90-degree pulse do not have exactly the same rates of precession. Because some precess faster than others, they spread out over time, and $M_{T}$, the transverse component of their sum, becomes smaller and eventually goes to zero.

The spin-echo pulse sequence involves an initial 90-degree pulse. Immediately following this pulse, the individual nuclear magnetic moments begin to relax back toward their longitudinal, equilibrium orientation, and, also, to lose precessional phase with each other. After an operator-selected time interval equal to one half of the time of echo (TE), a 180-degree RF pulse is given. This has the effect of reflecting the spread of nuclear magnetic moments from phase loss so that, after an equivalent time $1 / 2$ TE, a brief rephasing occurs. This rephasing produces a transiently increased emitted $\mathrm{RF}$ signal called an echo. TE is the time interval between the initial 90 -degree pulse and the echo. The strength of the echo depends on the selected interval of TE and on the rate of $\mathrm{T}_{2}$ dephasing in the tissue(s) being imaged.

Repeated measurements must be made to construct an MRI image. The time interval between the initial RF pulse of one pulse sequence and the initial pulse of the subsequent sequence is called the time of repetition (TR). The amount of transverse magnetization following the initial 90-degree $\mathrm{SE}$ pulse is equal to the amount of longitudinal magnetization just prior to the pulse. During repeated measurements, the amount of longitudinal magnetization available to be flipped by the initial 90-degree pulse of the next SE pulse sequence depends upon the amount of $\mathrm{T}_{1}$ relaxation that has occurred since the previous 90 -degree pulse. For example, if TR is much shorter than $\mathrm{T}_{1}$, very little $\mathrm{T}_{1}$ relaxation will be allowed between pulse sequences.

The MR signal intensity from point to point in an image depends on TR, TE, $\mathrm{T}_{1}, \mathrm{~T}_{2}$, and $\mathrm{D}$. The multiple parameters affecting image intensity make MRI complicated, but also very powerful. By varying $\mathrm{TR}$ and $\mathrm{TE}$, different images of the same tissue may be obtained, and the image contrast (differential appearance) of differing tissue types can be optimized. When TR and TE are short, image appearance is dependent most strongly on $\mathrm{T}_{1}$ values $\left(\mathrm{T}_{1}\right.$-weighted image). When $\mathrm{TR}$ and $\mathrm{TE}$ are long, image appearance is most strongly dependent on $\mathrm{T}_{2}$ values $\left(\mathrm{T}_{2}\right.$-weighted image). When TR is long but TE is short, image appearance depends more on tissue density.

\section{Imaging}

Fig 6 is a $T_{1}$-weighted image. The shorter the $T_{1}$ of a tissue, the brighter the image, because by having a short $T_{1}$, a tissue rapidly regains longitudinal magnetization and, therefore, is able to produce a larger magnetization in the transverse plane after the subsequent 90-degree pulse. Fat, blood in the methemoglobin state, and mucus have short $\mathrm{T}_{1} \mathrm{~s}$, and, therefore, they image brightly.

Fig 7 is a $T_{2}$-weighted image. Intensity of tissue in this image is a complex function of both the $T_{1}$ and $\mathrm{T}_{2}$ of tissue. In general, however, the longer the $\mathrm{T}_{2}$ of the tissue, the more intensely it appears in such images. CSF is intense on $\mathrm{T}_{2}$-weighted images because of its exceptionally long $T_{2}$.

Certain substances are black (signal void) on images produced by any pulse sequence. These include calcium, air, flowing blood (except in special circumstances), and hemosiderin found in resolving clotted blood. Calcium and air have a low signal intensity because they lack the relative number of hydrogen nuclei necessary to produce a signal observable by MRI.

Flow of blood and CSF is interesting. When flow is slow, the flowing substance may appear bright, but when flow is fast or turbulent, the substance appears dark. These properties of flow can be used in identifying aneurysms and arteriovenous malformations.

Blood clots in various stages of development or resolution (oxyhemoglobin, deoxyhemoglobin, methemoglobin, hemosiderin) have different intensities on $\mathrm{T}_{1}$ - and $\mathrm{T}_{2}$-weighted images. Hemosiderin demonstrates a signal void because of magnetic properties beyond the scope of this discussion. It was thought initially that extraluminal blood was visualized poorly by MRI. However, with the stronger magnetic fields now available for imaging, blood is well demonstrated, and the chronicity of its presence can be determined by these characteristics. ${ }^{1}$

Most pathologic conditions lead to damage of the cell membrane and the ATPase pump and cause accumulation of fluid. Increased fluid causes the 
pathologic region to have slightly decreased intensity on $\mathrm{T}_{1}$-weighted images and very increased intensity on $\mathrm{T}_{2}$-weighted images. This property allows MR (especially $\mathrm{SE}$ images, which are $\mathrm{T}_{2}$ weighted) to be very sensitive to the presence of a pathologic state, although it is not as specific for the type of pathologic conditions as once hoped.

\section{Contrast agents}

An advantage of MRI is that intravenous contrast agents are not needed to demonstrate a pathologic area, because of MRI's inherent contrast sensitivity. However, contrast agents such as gadoliniumDTPA are being developed and tested to enhance anatomic differentiation (for example, identifying bowel), enhance lesion detectability, and decrease the time required for an examination.

For example, meningiomas presently are difficult to see because they produce a signal similar to that of normal brain tissue. With the use of contrast medium, however, these hypervascular lesions are demonstrated easily. Also, $\mathrm{T}_{1}$-weighted images take less time to produce than $\mathrm{T}_{2}$-weighted images, but lesion contrast on $\mathrm{T}_{1}$-weighted images is not as great. Contrast agents may increase lesion detectability and specificity on the shorter $\mathrm{T}_{1}$ weighted images, and, therefore, they may shorten examination time.

\section{Spectroscopy}

As mentioned earlier, the resonance frequency of a specific element is dependent on the strength of the local magnetic field in which the nuclei exist. In fact, because of the nonuniform distribution of molecular electron clouds, the differential magnetic screening effect produces measurably different resonance frequencies for the nuclei of atoms bound at different molecular positions. This provides the capability of nuclear magnetic resonance spectroscopy. For example, spectral analysis of the nuclear magnetic resonance signal from ${ }^{31} \mathrm{P}$ nuclei bound in adenosine triphosphate (ATP) would reveal three peaks, each at a different frequency. Each of these peaks in the phosphorus spectrum results from the unique local magnetic field experienced by the phosphorus nuclei in the three different binding sites of the ATP molecule.

The nuclei of biomedical interest that are amenable to study by nuclear magnetic resonance spectroscopy include hydrogen, phosphorus, sodium, carbon, and fluorine. Most biomedical sampling is performed using hydrogen or phosphorus, because both elements provide reasonably strong nuclear magnetic resonance signals as well as data of biomedical interest. For example, the phosphorus spectrum provides a direct probe of metabolic status by meas-

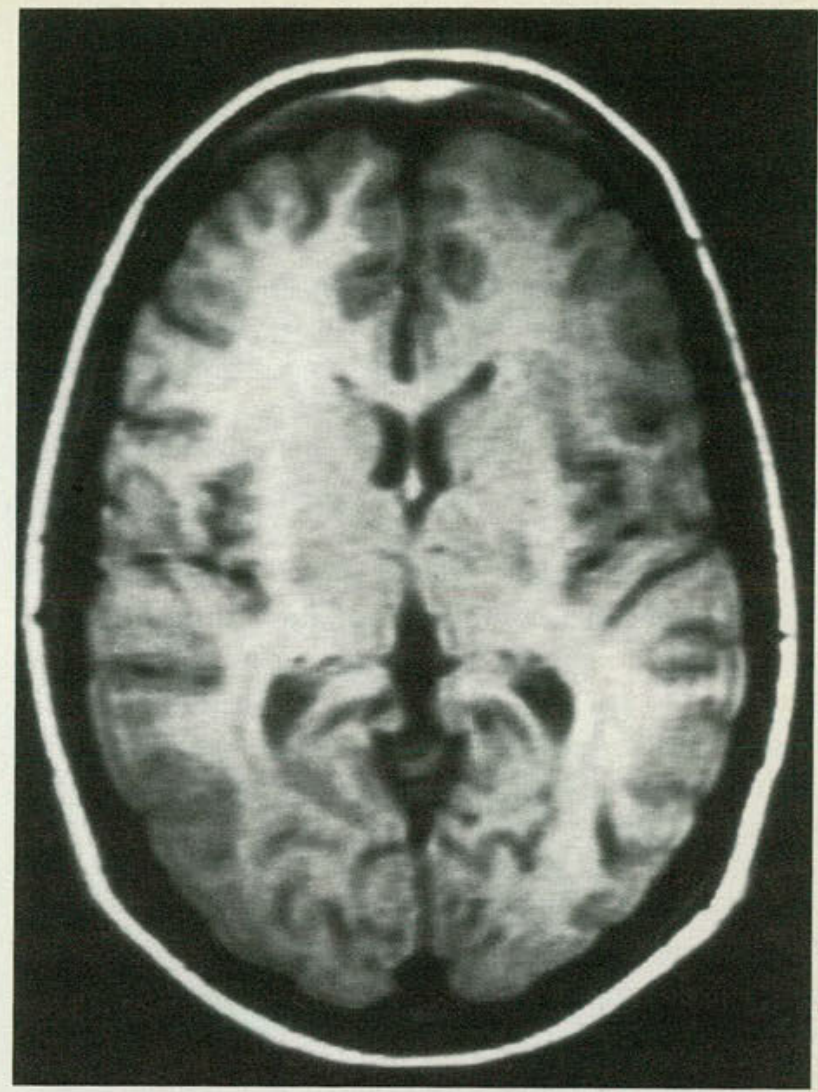

Fig 6. $T_{1}$-weighted magnetic resonance image of a normal brain.

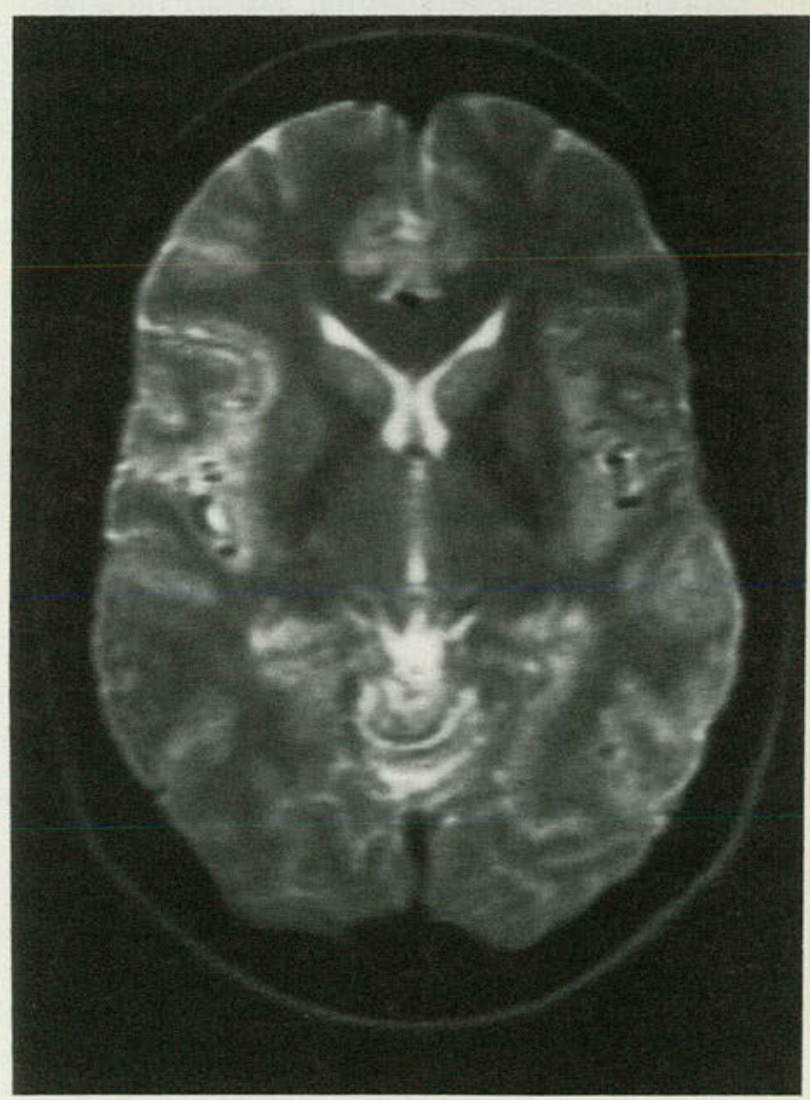

Fig 7. $T_{2}$-weighted magnetic resonance image of the normal brain. 
uring the relative quantities of phosphated compounds and inorganic phosphorus involved in the basic biologic energy exchange processes. When the dominant water and lipid peaks are suppressed, hydrogen spectroscopy may be used to investigate the wide array of hydrogen bearing compounds.

Nuclear magnetic resonance spectroscopy has been used as an in vitro laboratory technology for basic biologic and chemical research for many years. One may observe the presence and measure the relative concentrations of various biochemical analytes in a noninvasive, nondestructive manner. Reasonable potential currently exists to combine diagnostic clinical imaging and localized in vivo spectroscopy. This combination may enable, for example, in vivo tissue characterization (the noninvasive biopsy), or assessment of the distribution, degree, and effect on tissue viability resulting from ischemic insult to the brain, myocardium, or transplanted organ.

\section{Equipment}

Three types of magnets-superconductive electromagnets, resistive electromagnets, and permanent magnets-are available for the production of the main applied magnetic field. There are advantages and disadvantages to each type. Superconductive magnets can produce very strong, exceptionally stable, homogenous fields, which provide superior signal-to-noise ratio, spatial resolution, and tissue contrast. However, these magnets are expensive (system prices range from $\$ 1.4$ million to $\$ 2.2$ million), and they require costly liquid helium and liquid nitrogen to keep the magnet wires at superconductive temperatures (where no power is required to maintain the field). Fields up to $2.0 \mathrm{~T}$ have been achieved in large-bore (that is, whole-body) magnet systems ( $1 \mathrm{~T}=10,000$ Gauss $(\mathrm{G})$; the magnetic field of the earth averages about $0.5 \mathrm{G}$ ).

Resistive magnets may be used to produce fields of up to about $0.2 \mathrm{~T}$. Resistive magnets are less expensive, with system prices typically in the range of $\$ 0.8$ million to $\$ 1.3$ million. However, electric power consumption is great and actually can exceed the annual cost of liquid gases used by a superconductive magnet. Furthermore, resistive magnets produce a great deal of heat, which must be dissipated carefully by water cooling because field uniformity and stability critically depend on magnet temperature. The lower strength and diminished uniformity of resistive magnet fields results in diminished spatial resolution and image quality.

Permanent magnets are made from magnetic iron or ceramics; they can produce fields up to 4.3 Testa (T). Costing slightly less than resistive mag- net systems, they are the least expensive. Permanent magnets are free from mechanical failure, and operating costs are small. However, the weight of the magnet system can approximate 100 tons, which presents structural challenges, and their fields also are less uniform than superconductive magnet fields, which results in diminished spatial resolution and image quality.

\section{Biologic effects and safety}

The most important aspect of MRI safety is that it does not employ ionizing radiation. This fact alone makes it considerably safer than roentgenographic or nuclear medicine imaging procedures. The inherent contrast of tissues in MRI also obviates the need for injected or ingested contrast agents for most clinical applications.

When undergoing an MRI examination, the patient is exposed to a strong static magnetic field, time-varying gradient magnetic fields, and pulsed RF fields. Considerable data are available about occupational exposure to similar conditions by physicists and technicians working in particle accelerator laboratories. Furthermore, the potential adverse biologic effects of such conditions have been the subject of intensive research during the development of clinical MRI technology. This occupational experience and research have produced no evidence of adverse biologic effects, nor have any adverse effects been reported from actual clinical experience with the technology; the current level of clinical experience includes more than 1 million imaging procedures performed during the past decade..$^{2-5}$

Based on this experience and data, the Food and Drug Administration has established conservative guidelines for the use of MRI with "nonsignificant risk." All diagnostic imaging systems are operated within these guidelines.

Although no evidence exists which suggests MRI could be dangerous to pregnant women or fetuses, it is the policy of many MRI services to avoid imaging women who are suspected or known to be in their first trimester of pregnancy.

The magnetic fields employed in MRI can exert torsional forces on ferromagnetic objects. Modern asynchronous pacemakers are designed to begin cardiac pacing when exposed to magnetic fields of $\geqslant$ $15 \mathrm{G}(0.0015 \mathrm{~T})$. Therefore, patients with cardiac pacemakers are not candidates for MRI scanning. Similarly, other electromechanical devices, such as neurostimulators, will fail to function in a strong magnetic field, and MRI scanning also is contraindicated for patients with these devices. ${ }^{16}$

Hemostatic clips and surgical staples generally are safely secured by scar tissue approximately six 


\section{EFFICACY...SAFETY...EXPERIENCE}

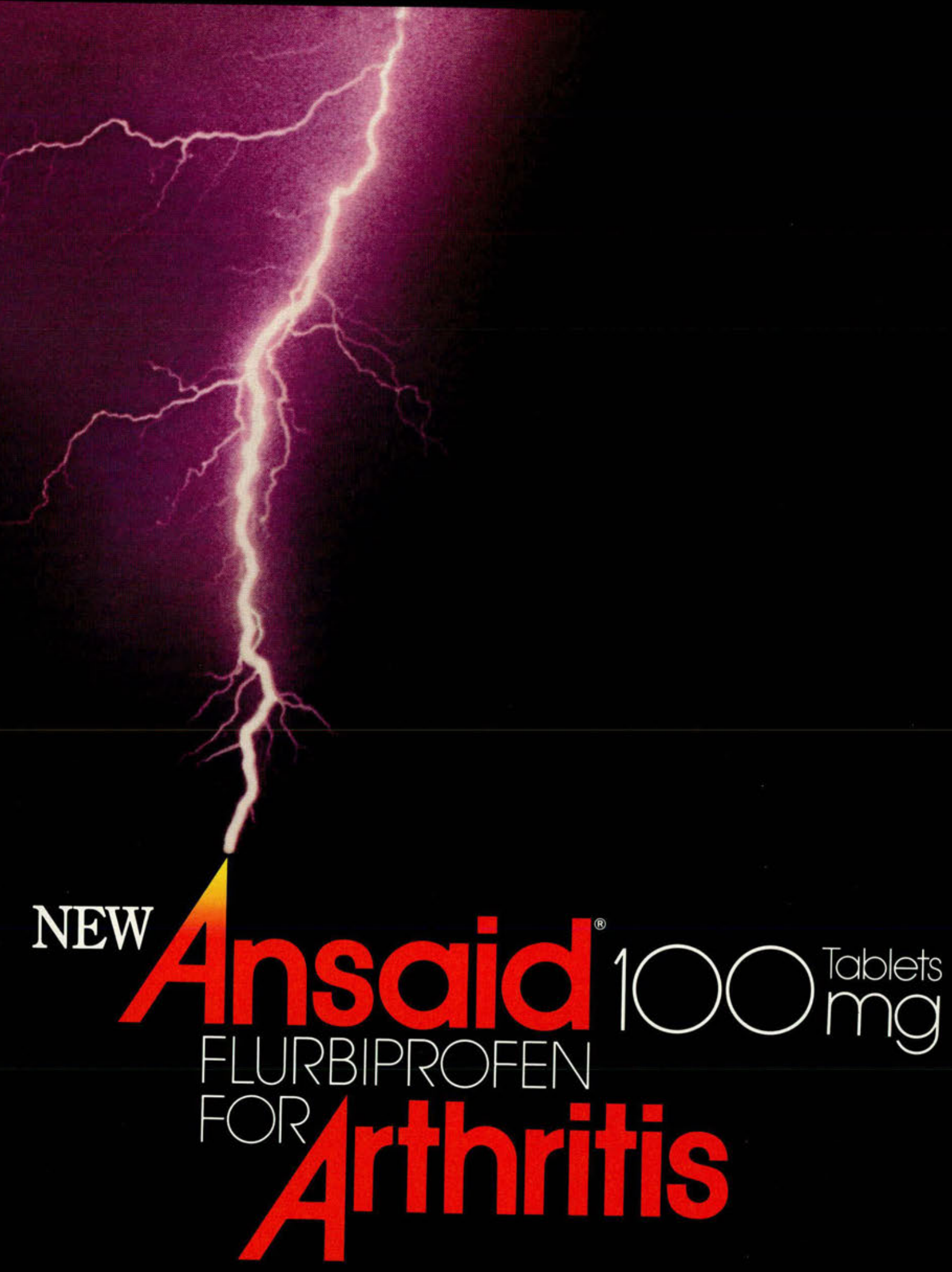




\section{AnsaidiOOmo: 1 Tablet BID or TID}

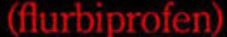 \\ for Osteoarthritis}

\section{Ansaid Tablets lessen}

disability in osteoarthritis

Reduction of disability (investigators' assessment) in a 12-week, double-blind, randomized study of 147 patients with osteoarthritis of the knee treated with Ansaid, up to $200 \mathrm{mg} /$ day or aspirin, up to $4,000 \mathrm{mg} /$ day. ${ }^{1}$

\section{Percent patients improved}

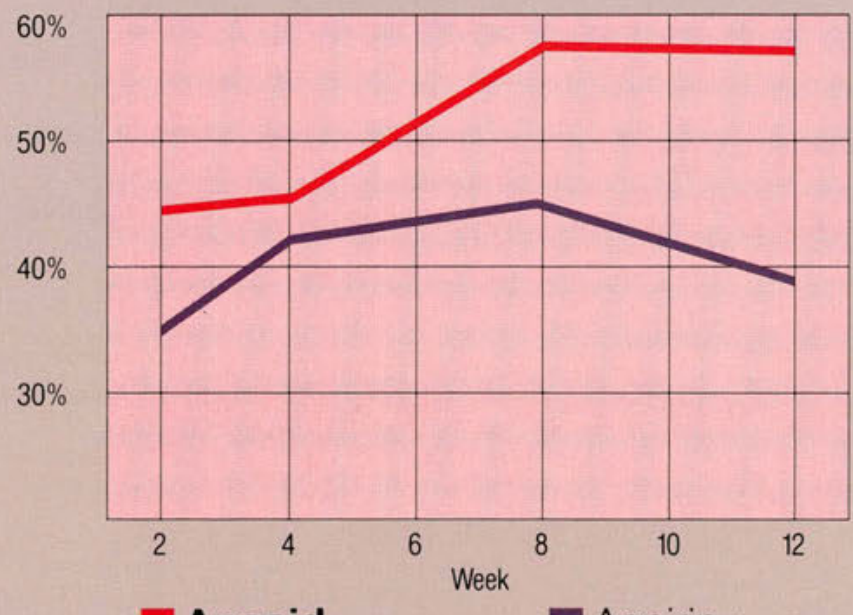

Ansaid $(n=63)$
Ansaid (flurbiprofen) “... is an effective drug in controlling the pain and other symptoms of osteoarthritis of the knee." ${ }^{1}$

\section{Convenient Dosage}

The dosage of Ansaid tablets is $200 \mathrm{mg}$ or $300 \mathrm{mg}$ daily, administered BID or TID. (Most experience in RA has been with TID or QID dosage.) Recommended starting dose is $100 \mathrm{mg}$ BID.

\section{Efficacy maintained week after week}




\section{EFFICACY, SAFETY, EXPERIENCE...}

\section{An excellent safety record worldwide}

- A safety profile derived from 4,123 patients who received flurbiprofen.

- 948 of these patients were treated for at least 6 months.

Incidence of side effects in three body systems during clinical trials with Ansaid

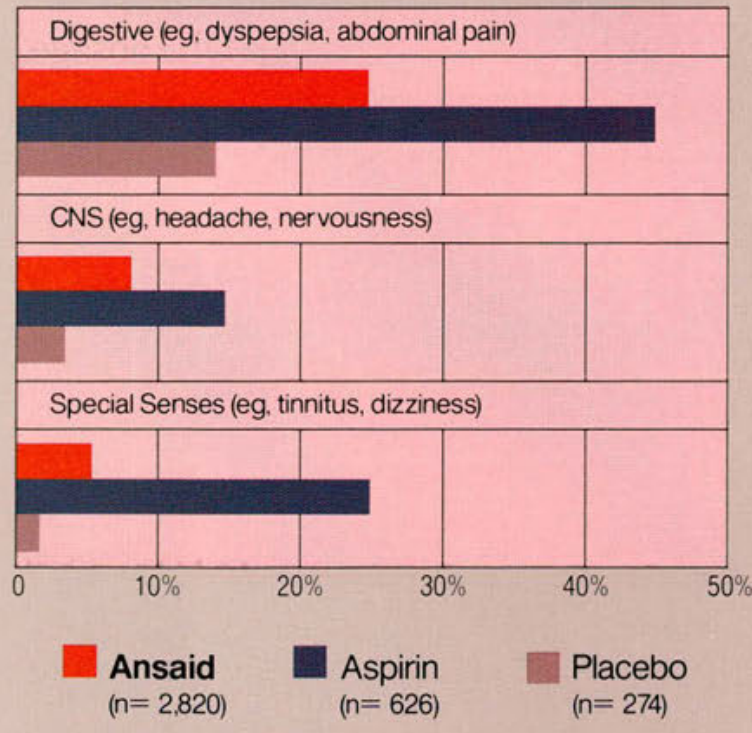

\section{Upjohn}

The Upjohn Company

Kalamazoo, MI 49001, USA

For a brief summary of prescribing information, please see the last page of this announcement.
- Overall incidence of side effects with Ansaid is about half that reported with comparable dosages of aspirin.

- Does not accumulate...excretion virtually complete 24 hours after the last dose.

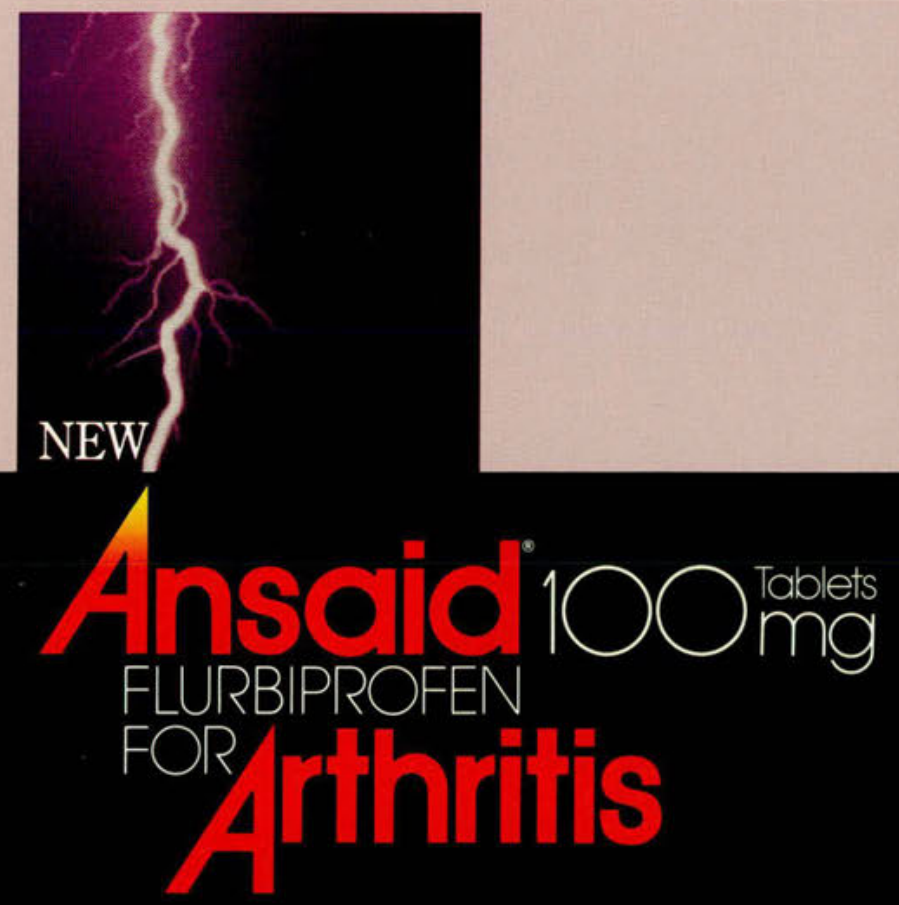




\section{Ansaid $100 \mathrm{mg}: 1$ Tablet BID or TID}

\section{for Rheumatoid Arthritis}

Ansaid Tablets: Effective in acute and chronic phases

Results of a 52-week, double-blind, randomized study of 822 patients in the United States, demonstrated that Ansaid 200 to $300 \mathrm{mg} /$ day relieves rheumatoid arthritis pain and inflammation as effectively as comparable dosages of aspirin.

\section{Convenient Dosage}

- The dosage of Ansaid tablets is $200 \mathrm{mg}$ or $300 \mathrm{mg}$ daily, administered BID or TID. (Most experience in RA has been with TID or QID dosage.)

Recommended starting dose is $100 \mathrm{mg}$ BID.

- Results of a 12-week study of 143 patients with rheumatoid arthritis comparing BID and QID dosing of Ansaid (flurbiprofen) $200 \mathrm{mg}$ daily indicated the BID dosing of Ansaid is as effective as QID dosing?
Improvement in efficacy parameters

Mean percent change from baseline

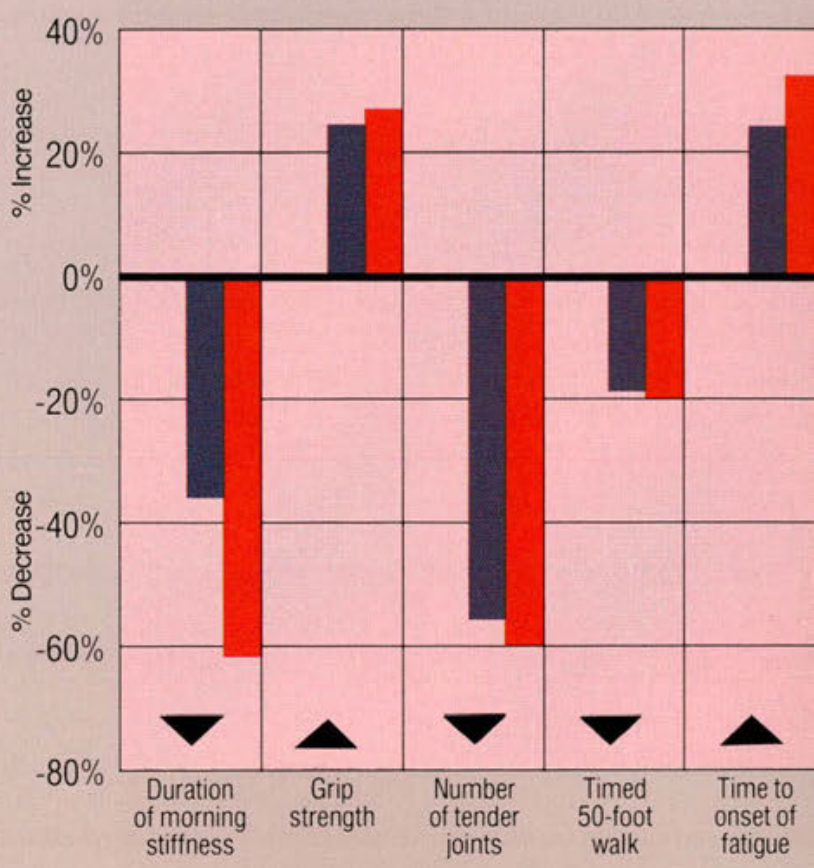

Aspirin ( $n=421)$ Ansaid (flurbiprofen) $(n=401)$

Effective...improves patient mobility. 


\section{EFFICACY, \\ SAFETY, \\ EXPERIENCE...}

\section{Over a decade of worldwide experience}

- Experience in 70 countries

- For over a decade, flurbiprofen has been a widely used antiarthritic agent.

- Since 1977 , there have been more than 1 billion patient-days of treatment with flurbiprofen throughout the world.

- An 8-year study involving 1,396 patients in the United Kingdom confirms the efficacy and safety of flurbiprofen in arthritis. ${ }^{3-5}$

- A multicenter study of 11,500 arthritic patients in France provides additional confirmation of flurbiprofen efficacy and safety. ${ }^{6}$

- In the United States, controlled trials involving 2,820 patients further demonstrated the clinical efficacy and safety of Ansaid (flurbiprofen).
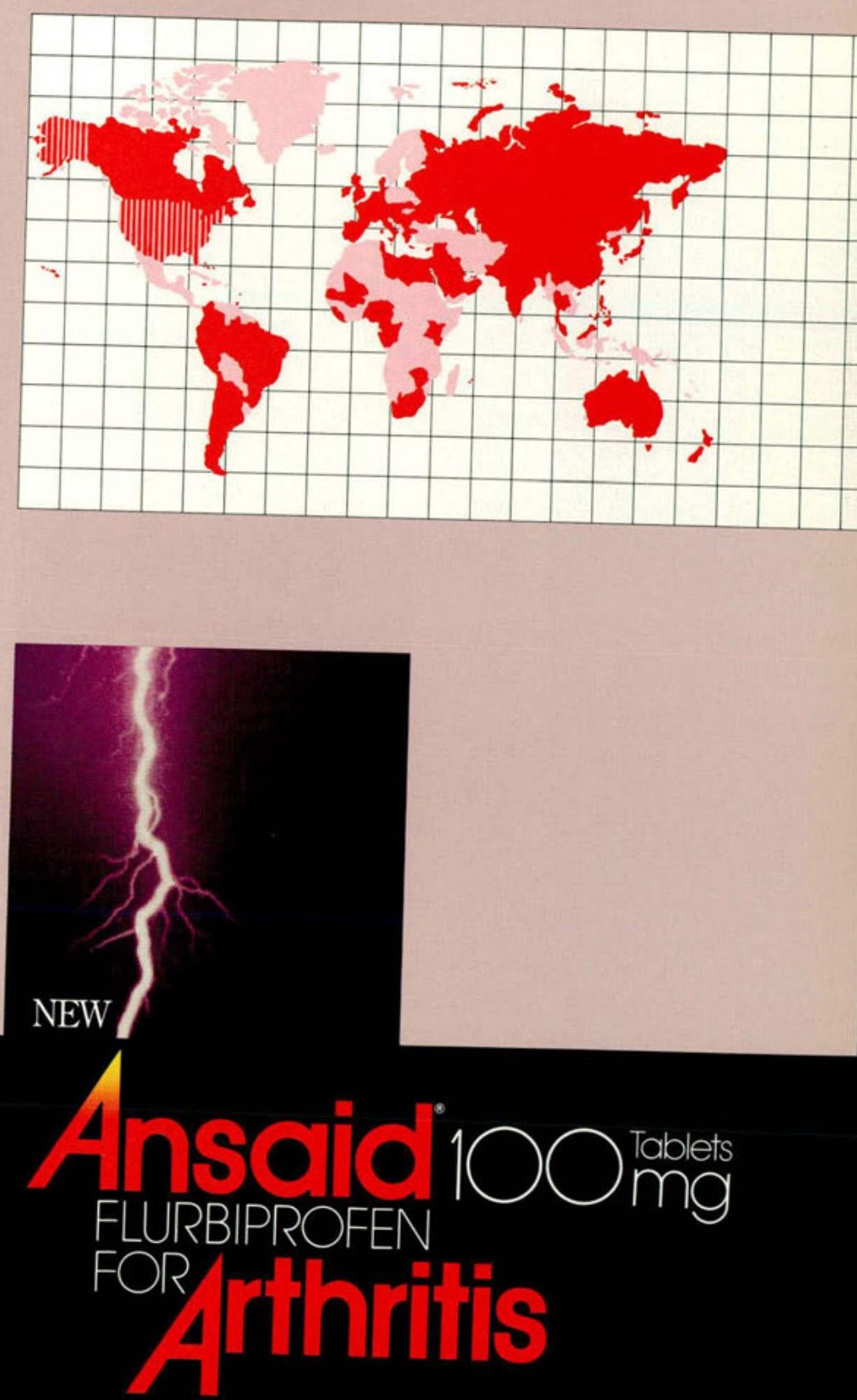


\section{EFFICACY, SAFETY, EXPERIENCE...}

The dosage of Ansaid tablets is

$200 \mathrm{mg}$ or $300 \mathrm{mg}$ daily, administered

BID or TID. (Most experience in

RA has been with TID or QID dosage.)

Recommended starting dose is $100 \mathrm{mg}$ BID.

\section{ANSAID ${ }^{\circledR}$ Tablets}

INDICATIONS: Acute and long term treatment of signs and symptoms of rheumatoid arthritis and osteoarthritis. CONTRAINDICATIONS: Hypersensitivity to ANSAID, or if aspirin or any other nonsteroidal anti-inflammatory agent induces asthma, urticaria or other allergic type reactions. Fatal asthmatic reactions have been reported in such patients. WARNINGS: Gastrointestinal effects: Risk of GI ulcerations, bleeding and perforation with nonsteroidal anti-inflammatory therapy: Serious GI toxicity can occur at any time, with or without warning symptoms, during chronic treatment. The occurrence is about 1\% after 3-6 months, 2-4\% after a year. Patients should be informed of signs and symptoms of serious GI toxicity and what to do if it occurs. No subset of patients not at risk has been identified. Prior history of serious Gl events and other risk factors of peptic ulcer disease, e.g. alcoholism, smoking, etc. have been associated with increased risk. The elderly and debilitated tolerate ulceration and bleeding less well. Higher doses probably carry a greater risk. Gl ulceration and bleeding can occur without warning symptoms and chronically treated patients should be followed. PRECAUTIONS: Patients with impaired renal or hepatic function: Use ANSAID and similar agents cautiously. Pharmacokinetics have not been studied in patients with decreased liver function. Renal Effects: Rats develop renal papillary necrosis at dosages equivalent to human therapeutic levels, as do monkeys given 20-40 times the human dose. In clinical studies of ANSAID, kidney function tests were done monthly and renal effects were similar to those seen with other nonsteroidal anti-inflammatory drugs. A second form of renal toxicity has been seen in patients with prerenal conditions that reduce renal blood flow or blood volume. A nonsteroidal anti-inflammatory drug may cause dose-dependent reduction in prostaglandin formation and precipitate overt renal decompensation. Patients at greatest risk are those with impaired renal or hepatic function, heart failure, those taking diuretics or the elderly. Drug discontinuation usually leads to recovery. Patients at high risk on chronic treatment should have renal function monitored if they have signs or symptoms that may be consistent with mild azotemia, e.g. malaise, fatigue, loss of appetite. Occasionally BUN and serum creatinine may be elevated without signs or symptoms. Flurbiprofen is excreted by the kidneys and pharmacokinetics are changed by renal failure so patients with renal failure should be monitored and may require a reduction of dosage to avoid accumulation of flurbiprofen metabolites. Liver tests: Borderline elevations of liver function tests may occur in up to 15\% of patients, and may progress, remain unchanged or disappear with continued treatment. Patients with signs and/or symptoms or with an abnormal liver function test should be evaluated further. Anemia: Patients treated long term who have initial hemoglobin values under $10 \mathrm{~g} / \mathrm{dL}$, should have periodic hemoglobin values. Fluid retention and edema: Fluid retention and edema have been reported so use ANSAID with caution in patients with conditions such as cardiac decompensation or hypertension. Vision Changes: Blurred and/or diminished vision has been reported. Patients with eye complaints should have periodic ophthalmologic exams. Effect on platelets and coagulation: Platelet aggregation is inhibited and bleeding time prolonged; patients who may be adversely affected should be carefully observed. Information for patients: Physicians and patients may wish to discuss potential risks and likely benefits. Drug Interactions: Anticoagulants: Bleeding parameters are affected, clinical bleeding has been reported. Aspirin: Flurbiprofen levels were $50 \%$ lower. Concurrent use is not recommended. Betaadrenergic Blockers: Pharmacokinetics and heart rate reduction are not affected, hypotensive effect of propranolol but not atenolol was attenuated. Cimetidine, Ranitidine: Cimetidine causes a 13\% increase in area under the flurbiprofen serum concentration curve. Diuretics: Patients receiving furosemide or thiazides should be closely observed to make sure the desired effect is obtained.

References

1. Lomen PL, Lamborn KR, Porter GH, et al: Treatment of osteoarthritis of the knee: A comparison of flurbiprofen and aspirin. Am JMed 1986; 80(suppl 3A): 97-102.

2. Brown BL, Daenzer CL. Hearron MS, et al: Comparison of two dosing schedules of flurbiprofen for patients with rheumatoid arthritis. Am JMed 1986; 80(suppl 3A): 19-22. 3. Busson M: A long-term study of flurbiprofen in rheumatological disorders: I. Rheumatoid arthritis. JInt Med Res 1986:14:1-6.

4. Busson M: A long-term study of flurbiprofen in rheumatological disorders: II. Osteoarthritis. JInt Med Res 1986;14:7-12.

5. Busson M: A long-term study of flurbiprofen in rheumatological disorders: III. Other articular conditions.

JInt Med Res 1986;14:13-18.

6. Kieffer G: Flurbiprofen: A multicenter trial in 11,500 ambulatory patients (French). Rev Med 1981;22:915-924.
Carcinogenesis, mutagenesis, impairment of fertility: No evidence. Teratogenic effects: Pregnancy category B: No effect in animals. Not recommended for use in pregnancy. Labor and delivery, nursing mothers, pediatric use: Use is not recommended. ADVERSE REACTIONS: $9.4 \%$ of 4123 patients dropped out of studies because of an a.d.r. Incidence $>1 \%$ : Gastrointestinal: Dyspepsia*, diarrhea*, abdominal pain*, nausea*, constipation, G bleeding, flatulence, elevated liver enzymes and vomiting. Central nervous system: Headache* "stimulation" (e.g. anxiety, insomnia, reflexes increased, tremor) and "inhibition" (e.g., amnesia, asthenia, somnolence, malaise and depression). Respiratory: Rhinitis. Dermatologic: Rash. Special senses: Dizziness, tinnitus and changes in vision. Genitourinary: Signs and symptoms suggesting a urinary tract infection* Body as a whole: Edema* Metabolic/nutritional: Body weight changes. *Reaction in 3 to $7 \%$ of patients. Incidence $<1 \%$ (Causal relationship probable): Gastrointestinal: Peptic ulcer disease (See Warnings), gastritis, bloody diarrhea. stomatitis, esophageal disease, hematemesis and hepatitis, cholestatic and non-cholestatic jaundice. Centralnervous system: Ataxia, cerebrovascular ischemia, confusion, paresthesia and twitching. Hematologic: Decrease in hemoglobin and hematocrit, iron eosinophilia and ecchymosis, thrombocytopenia, hemolytic anemia and aplastic anemia. (See Precautions) Respiratory: leukopenia, anemia, deficiency Asthma and epistaxis. Dermatologic: Angioedema, urticaria eczema and pruritus: photosensitivity, toxic epidermal necrolysis and exfoliative dermatitis Special senses: Coniunctivitis and parosmia. Genitourinary: Hematuria and impairment of rena function, interstitial nephritis. Body as a whole: Anaphylactic reactions, chills, fever. Metabolic, Nutritional: Hyperuricemia. Cardiovascular: Heart failure, hypertension, vascular disease and vasodilatation Incidence $<1 \%$ (Causal relationship unknown): Gastrointestinal: Periodonta abscess, appetite changes cholecystitis and dry mouth. CNS. Convulsion meningitis, hypertonia, cerebrovascular accident, emotional lability and subarachnoid hemorrhage. Hematologic Lymphadenopathy. Respiratory: Bronchitis, laryngitis, dyspnea, pulmonary embolism, pulmonary infarct. hyperventilation. Dermatologic: Alopecia, nail disorder, herpes, dry skin anc sweating Special senses: Ear disease corneal opacity glaucoma retrobulbar neuritis, change in taste, transient hearing loss, retinal hemorrhage. Genitourinary: Menstrual disturbances, vagina and uterine hemorrhage. vulvovaginitis, prostate disease. Metabolic nutritional: Hyperkalemia Cardiovascular: Arrhythmias, angina pectoris and myocardial infarction AGE AND ADMINISTRATION: 20 to 300 ma daily, administered bid, tic or qid. (Most experience in theuma toid arthritis has been with tid or qic dosage). Dose should be tailored to severity of symptoms and patien response. Store at controlled room temperature $\left(15-30^{\circ} \mathrm{C}\right)$. Federal lav prohibits dispensing without prescription.

\section{Upjohn}

The Upjohn Company Kalamazoo, MI 49001, USA

NEW .

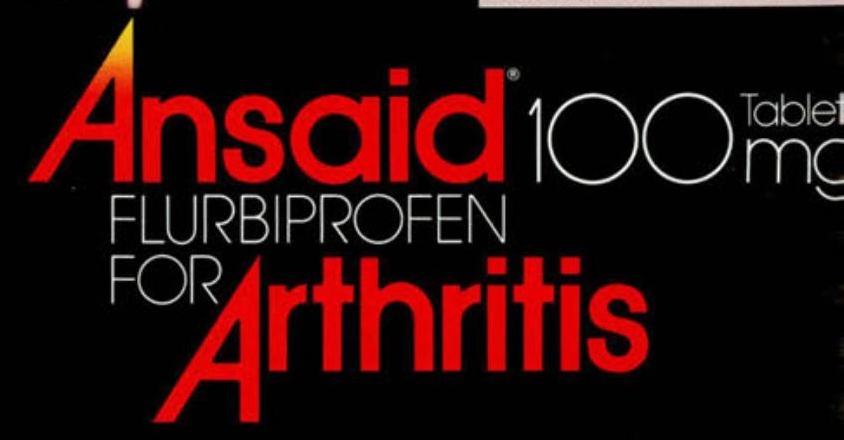


months after surgery. Prior to that time, MRI imaging should be used with care. Similarly, patients with a history of a possible metallic foreign body in the eye or brain generally are not candidates for MRI scanning, or they should be scanned only after careful preprocedure screening. Patients with most prosthetic heart valves may be safely scanned, with the exception of the Starr-Edwards Pre-6000 valve. ${ }^{17}$

Other metal implants and prostheses generally do not present problems for MRI scanning, except that image artifacts may be caused by the effect of ferromagnetic implants on the local magnetic field. More recent implants are being produced with less ferromagnetic properties and tend to minimize these effects.

The static magnetic field produced by most clinical MRI imagers is very strong; it generally extends well beyond the physical dimensions of the magnet. A significant danger exists in the possibility of loose ferromagnetic objects in the vicinity of the magnet system being accelerated as missiles toward the magnet. Thus, it is important that access to the magnet room be controlled very carefully. A patient undergoing an MRI procedure who requires emergency medical care or resuscitation first must be removed from the magnet room to avoid inadvertent careless use of ferromagnetic objects, and also because most resuscitation and monitoring equipment does not operate properly in a strong magnetic field.

\section{Factors affecting image quality}

Because motion severely degrades a magnetic resonance image, patients who cannot remain still or cannot be sedated are not candidates for MRI. A small number of patients cannot start or complete an examination because of the claustrophobia they experience when placed in the magnet bore. Often these difficulties can be overcome by mild sedation and a nonthreatening environment.

As mentioned previously, some metallic objects may prevent an adequate examination because of artifacts they produce in the image. This degrada- tion of the image generally is quite local to the object, and it is more a factor of the ferromagnetic properties of the material than its overall size.

\section{Conclusions}

The physics of MRI is complicated. However, not all of the nuances have to be understood completely to be able to gain information from magnetic resonance images or to appreciate MRI's capabilities and limitations. MRI is an exceptionally safe procedure and provides superior spatial resolution and tissue contrast. This modality provides the radiologist and the general medical community with an exciting new procedure to evaluate pathologic states. It already has and will continue to revolutionize diagnostic medicine.

\footnotetext{
1. Gomori JM, Grossman RI, Goldberg HI, et al: Intracranial hematomas: Imaging by high-field MR. Radiology 1985;157:87-93.

2. Budinger TF, Cullander C: Health hazards in nuclear magnetic resonance in vivo studies. Radiographics 1984;4:74.

3. Biological effect of MRI: A clean safety record so far. Diagn Imaging 1987;9:96-101.

4. Thomas A, Morris PG: Effects of NMR exposure on living organisms: I. A microbial assay, abstract. Radiology 1982;143:310.

5. Cooke P, Morris PG. Effect of NMR exposure on living organisms: II. A genetic study of human lymphocytes, abstract. Radiology 1982;143:310.

6. Pavlicek W, Geisinger M, Castle L, et al: Effect of nuclear magnetic resonance on patients with cardiac pacemakers, abstract. Radiology $1983 ; 147: 149$

7. Soulen RL, Budinger TF, Higgins CB: Magnetic resonance imaging of prosthetic heart valves. Radiology 1985;154:705-707.

Harms SE, Morgan TJ, Yamanashi WS: Principles of nuclear magnetic resonance imaging. Radiographics 1984;4:26-43

Mitchell MR, Tarr RW, Conturo TE: Basic principles for choosing MRI pulse sequence timing intervals. Radiographics 1986;2:245-260.

Morgan TJ, Hendee WR: Introduction to Magnetic Resonance Imaging. Denver, Multimedia Publishing, Inc, 1984, pp 50-52.

Partain CL, Price RR, Patton JA, et al: Nuclear magnetic resonance imaging. Radiographics 1984;4:5-25.

Wolfe GL, Popp C: NMR, A Primer for Medical Imaging. Philadelphia, Slack Publishing, 1984.
}

From the Department of Radiology, College of Osteopathic Medicine, Michigan State University, East Lansing, Mich.

Reprint requests to Dr Pera, B220 Clinical Center, MSU-COM, East Lansing, MI 48824. 


\section{D.F.M. Clinician: Section of O.P.P.}

The Ohio University College of Osteopathic Medicine is seeking a physician (D.O.) for a full-time, tenure-track, clinical faculty position in the Department of Family Medicine, Section of Osteopathic Principles and Practice. The successful candidate will have special interest and qualifications in the practice and teaching of osteopathic principles and practice.

\section{Responsibilities and Qualifications:}

1. Teach coursework assigned to the Department of Family Medicine with priority given to the teaching of osteopathic principles and practice.

2. Participate in ongoing programs of the Section of Osteopathic Principles and Practice including pre- and postdoctoral training, and research as appropriate.

3. Maintain, demonstrate, and teach the clinical application of osteopathic principles in ambulatory and hospital-based practice.

4. Be a graduate of an AOA-approved osteopathic college, have satisfactorily completed an AOA-approved internship, be Board-eligible or Board-certified in General/Family Practice by the AOBGP, or by the Board on Fellowship of the American Academy of Osteopathy.

5. See patients twelve (12) hours weekly in the Osteopathic Medical Center.

\section{Rank:}

Assistant/Associate Professor-commensurate with experience/credentials

\section{Salary:}

Base salary:Assistant Professor- $\$ 28,999-\$ 36,000$

Associate Professor- $\$ 36,000-\$ 42,000$

\section{PLUS}

Practice Plan salary: negotiated according to clinical capabilities and faculty service

Research facilities available. Rural environment with cultural benefits of university town. Excellent fringe benefits.

\section{Closing Date:}

September 1,1989 , or until filled

Ohio University is an Affirmative Action/Equal Opportunity Employer

Application may be made to: Anthony G. Chila, D.O., F.A.A.O.

Professor and Chairman, Department of Family Medicine

Chief of Clinical Research

Grosvenor Hall

Ohio University College of Osteopathic Medicine

Athens, Ohio 45701

PH: $614 / 593 / 2210$ 